\title{
A RESEARCH STRATEGY FOR INVESTIGATING BUSINESS PROCESS MANAGEMENT APPROACHES
}

\author{
James Gibson \\ Decision Systems Laboratory \\ University of Wollongong \\ NSW 2522 Australia \\ jgibson@uow.edu.au
}

\begin{abstract}
We are witnessing a revolution in industry which, if successful, will change forever how business systems are developed and the type of staff required. This paradigm shift has only recently become possible as business process conceptual understanding evolved, technologies have matured and higher abstraction levels have become possible.

Industry leads Business Processing Systems research as it has the strategic imperative and resources to be effective. Academic research is faced with three challenges: firstly, how to do effective research in an area of such broad scope, secondly, how to make research relevant to practice, thirdly how to spend limited resources effectively.

This paper defines the research framework for effective academic research at the University of Wollongong by the Software Effective Process group. Effective research is enabled by co-ordinating research based on the primacy of the business model and its resultant effective representation in executable systems. The framework aims to build a core research team, promote strong synergy with existing research areas, and create academic and industry relevant research.. We report on the results to date of our pilot program and seek feedback and advice to help us refine our approach.

A major Australian project is utilising a new software development lifecycle for 'system of systems' development which has arisen out of this research strategy. Later papers will report on both the theoretical basis and practical impacts of this work and other research by the group.
\end{abstract}

\section{INTRODUCTION}

Corporations have acted on the realisation that business process innovation and management provides uniqueness and the competitive edge in the marketplace. Implementing this is difficult, traditional systems development approaches carry high risk, are not scaleable, and are prone to failure. New ways of developing systems are arising with models and artefacts based upon business process as opposed to technical semantics, and founded on defined systems architectures. Some lifecycles target 'systems of systems' design with a pragmatic focus on issues of skills, staffing and scalability for large projects. Other lifecycles pursue the logical extension by creating business execution engines to directly execute the business semantics.

The I.T. industry has responded by extending lifecycles such as the Rational Unified Process, developing Business Process Management Systems (BPMS), notations such as BPMN, and related technologies (eg web services). Conceptually, the emphasis is now on process and process processing rather than data and data processing. Major processes are built up out of smaller atomic processes. Both major and atomic processes can be modified to keep track of changing business imperatives. The aim is to have the business processes endure for the life of the enterprise. It is the corporation and evolves as the corporation changes.

It is difficult to effectively evaluate both the new lifecycles, and their potentially executable forms. Not only must the ability to express process at the requisite detail be examined, but there are many other issues. Some of these issues are useability focussed, some test the 
suitability for use as mission critical systems, others involve legacy system integration, skill sets, scalability and maintainability. Multiple competing standards exist in various stages of completeness. For those lifecycles requiring executable engines, there is a plethora of vendor solutions and lack of underlying formal descriptions for most of these systems. Industry must consider their own unique circumstances, and choose between a traditional, or, an untested and potentially high risk executable approach.

Still it remains that these approaches must be evaluated, fundamentals understood, and possible guidelines for future evolution developed. Industry and Academia both have roles to play in the definition, implementation and evaluation of Business Process Systems (BPS) and related development lifecycles. As academic researchers, we have limited resources and staff but still want to make a worthwhile contribution. Focus and synergy is essential. We must apply triage to select tasks which have the best outcomes not only for the individual but also for the group.

In this paper, we present a research framework. This framework is intended to give us guidelines to choose between possible courses of action, and to best direct our efforts. The framework touches only lightly on the actual research areas, rather it is a set of rules for how to choose research and manage research, and build synergies both within academia and to industry. Such a framework is important as it provides an antidote for the short term, operational approach currently encouraged by Australian universities key performance indicators.

It is hoped that this research framework will give structure to long term, strategic research with the aim of promoting productivity, communication and interaction amongst researchers in this area with practice ${ }^{4}$ relevant outcomes. An earlier version of this paper was presented at the BPMDS Workshop at CAiSE'05, Portugal.

\section{BACKGROUND}

For the first fifty years, IT has been about the processing, storing and transporting of information in digital form.(Carr 2003) Technology drove the marketplace and solutions were localised and resistant to change. Businesses had the choice of either building custom applications or purchasing packaged products embodying fixed business processes such as ERP solutions. IT was thought of in terms of applications, hardware and network. Importantly, none of these approaches enabled long term strategic outcomes and change was damagingly expensive.

Now, a clear distinction is emerging between core and support processes. Support processes are those which every company requires and are not used for competition. For example, most companies do not compete on the effectiveness of their payroll system. Core processes implement the business strategies and provide the differentiators on which the corporations survival depends. Support processes, along with networking and hardware, are following the process of commoditisation.

This distinction is reflected in ongoing discussion as to the future of IT. Some papers, notably Nicholas Carr in an article in the Harvard Business Review entitled "IT doesn't matter", have generalised commoditisation occurring in support processes and drawn

\footnotetext{
${ }^{4}$ Crucially for us, how can we as IS researchers make a relevant practice based contribution? Zmud, R. (1998). "Editors Comments." MIS Quarterly 28 No. 3: pp. xxxix-xxxii.
} 
analogies to railroad and electricity utilities, claiming that it is now not possible to gain competitive advantage from IT.(Carr 2003). Other papers argue strongly that "the core functions of IT is process processing. This stance encourages business leaders to finally execute strategy innovations using agile, unique, proprietary (to them) and technologyenabled business processes that harness commoditised infrastructural transport and processing technologies."(Smith H. 2003)

Stark decisions must be made which can directly lead to the success or failure of corporations. The Gartner group in the book "The new CIO Leader"(Broadbent M. 2003) argues that Chief Information Officers will follow one of two paths based on these perspectives. One path leads to the static role of chief technology mechanic" the other to the role of new CIO leader. A chief technology mechanic can cut costs and 'hold the fort', a new CIO leader can drive business effectiveness and efficiency measures, and in breaking-away enterprises identify higher risk, long-term projects that offer possibilities for significant future competitive advantage.

\section{What is Business Process Management?}

Howard Smith and Peter Fingar in their book, Business Process Management- the Third wave, provide a succinct definition of the scope:

"Not only does Business Process Management encompass the discovery, design, deployment, execution, interaction, control, optimisation, and analysis of processes, but also the executive, administrative and supervisory control over them to ensure they remain compliant with business objectives for the delight of the customers. Processes are the main intellectual property and competitive differentiator manifest in all business activity."(Smith H 2003)

$\mathrm{BPM}^{5}$ (management) morphed out of BPM (modelling). Initially, corporations modelled their business processes as part of business process re-engineering.(Hammer and Champy 1993). This initial use of modelling failed for reasons identified by the Gartner Group as including: the effort required, poor prior experience of Computer Aided Software Engineering (CASE) tools, lack of time, difficulty mapping models to software artefacts, and the rate of business change causing models to fall out of synch.(Gartner 1997).

Two approaches grew out of this initial failure. The 'lifecycle' approach re-visited modelling with the aim of defining a more effective software development lifecycle and artefacts which would be effective in large systems building. The 'executable' approach saw Modelling morphed to management as the realisation came that the models could be executable. Models became executable and user interfaces, rather than being separate, became automatically generated from and by the process. Executable business processes and process data become the key artefact and are defined, managed, maintained and executed either automatically or manually.

Both approaches have only recently become possible as the weaknesses in existing lifecycles became apparent, business process conceptual understanding evolved, technologies have matured and higher abstraction levels have become possible. Theoretical underpinnings including $\mathrm{Pi}$ calculus have arisen out of over twenty years of computer science research(Milner), standards are being proposed by the Business Process

\footnotetext{
${ }^{5}$ An unfortunate choice of acronyms!
} 
Management Initiative organisation (BPMIorg) and Object Modelling Group (OMG), and applied R \& D is resulting in commercial products.

\section{THE FRAMEWORK}

The framework guides us in the difficult task of picking winners for our BPS research. It covers three areas:

- Choosing research.

- Attracting industry.

- Managing our research effort.

The framework consists of a set of statements. It is not a prescriptive document nor is it complete. Rather, it is a focus for ongoing discussion and iterative development. Statements are not set in concrete, if there is a competing consistent assertion with sufficient countering evidence then this will be adopted. The framework is not complete, it is a philosophy on how to conduct research in this area, it can be added to and changed as we make mistakes and learn, not a detailed definition of how this research will be conducted.

\section{CHOOSING RESEARCH AREAS.}

\section{Concentrate on business domain solutions}

Business strategy is about creating, re-creating and sustaining organizational forms that will enable a process of strategic response appropriate to life on the edge of chaos. What is important is the ability to create and effect new structures and processes quickly and at low cost.(Sauer 2003)

Traditional software engineering processes promote either agile or ceremonial development lifecycles in the technical domain. Almost all such processes encourage further requirements elicitation during technical implementation. The transformations between the business and technical domain cause unavoidable inefficiency and risk; and no business benefit. These processes are a historical legacy of a technology push approach to systems implementation. They are fundamentally flawed because they work in the wrong domain.

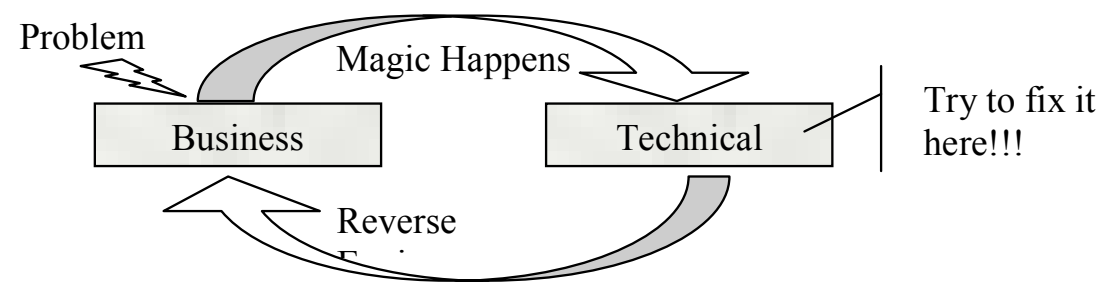

A Business Process paradigm demands that the solution be expressed in the business domain, not the technical domain. Lifecycle approaches can be thought of as choosing a 
business Process Oriented approach, as opposed to Object Oriented or Procedural. This creates different development artefacts, modelled at a higher level of abstraction and still requiring final technical implementation. Such approaches potentially provide immediate tangible benefits due to the new artefacts, plus an incremental pathway to later adoption of full executable systems if so desired. Executable systems themselves promise an order of magnitude increase in productivity by largely avoiding the technical domain.

Enterprise is wary of executable systems as they have no proven track record and it is hard to distinguish capability from marketing at this early stage. Improved business process understanding, maturity in systems frameworks and industry resolve promise success.

Statement: We will research the evolving business domain approaches as they promise the greatest benefit. Traditional software engineering approaches will only be used for comparison.

Concentrate on executable Process oriented solutions.

There are two business domain approaches. Development can be seen as the logical extension of existing coding languages, or as a totally new 'process oriented' language with its own conceptually different syntax and semantics running on a process execution engine.

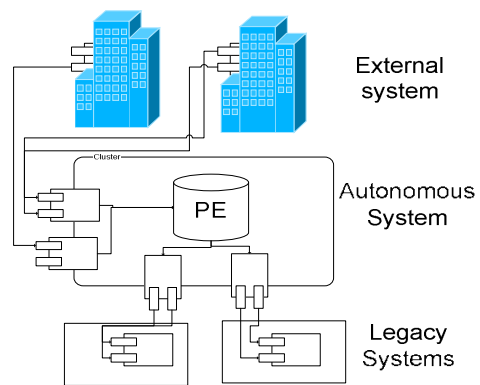

Process Engine Apvroach

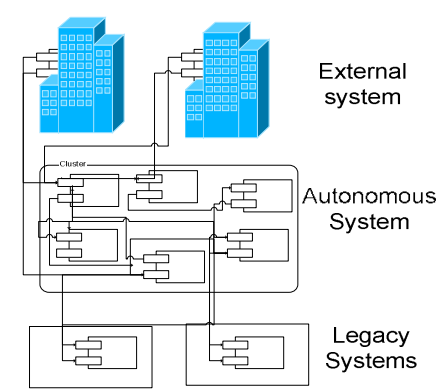

Coding Apvroach

Figure 1. Comparing Process Engine to Coding Approaches

A process engine executing a Process oriented language allows separation of domain versus system implementation. Technology no longer drives development. The process engine approach renders technology subservient and relegates it to being the glue binding autonomous and legacy systems. In contrast, a coding approach expresses business requirements in the technical domain and drives technical complexity throughout the solution. Process oriented approaches provide a compelling argument that they will be more productive, maintainable and agile than 'coding' solutions.

Statement: Our research will focus on Process--oriented business domain systems. Other approaches will form the basis for comparative studies. Apply theory to understand complexity

Process oriented approaches have a strong unifying paradigm. The paradigm underpins the development of execution engines, Process Query Languages, process activity monitors, and supporting CASE tools. This paradigm is based upon Pi calculus and Petri nets. It finds expression in the underlying software engineering meta-models and system executable 
architectures. Understanding this paradigm provides the analytic lever to express and understand not only Process oriented systems but also emerging web services and other standards.

Statement: We will manage complexity by evolving a consistent paradigm; which will guide our analysis.

\section{ATTRACTING INDUSTRY PARTICIPATION}

"In theory, there is no difference between theory and practice, In practice, there is",

Target national strategic objectives

The Australian government has identified areas of national strategic importance which includes supply chains and health. Strategic alliances in these application areas are required as we cannot address them by ourselves. The alliance must demonstrate a strong understanding of application area theory and issues. Our role in the alliance is to provide the theoretical horsepower and pragmatic capability to reason about and realise these applications.

Statement: Targeting national strategic objectives will stimulate research and make the decision making process by grant authorities as simple and attractive as possible.

\section{Identify suitable industries}

There are three types of industry involvement. Each can be addressed by different partners in the alliance:

- Application industries. Industry partners from customer systems. Examples being Transport companies, Health Care, or major manufacturers

- Solution seeking industries. More localised industry partners with a specific, usually decision based, problem. For example, trying to solve transport fleet allocation and res-scheduling.

- Process and Lifecycle. Software Engineering and implementation companies. Product vendors.

Statement: We will work with our alliance to put together a complementary set of industry partners.

\section{Provide tangible outcomes}

Industry partners want results, not just blue sky theories. We will provide the pragmatic software engineering capability to implement, deploy and test such systems.

Statement: We will provide tangible deliverables based on sound theory

\footnotetext{
${ }^{6}$ JAN L.A. VAN DE SNEPSCHEUT. Dutch-American computer scientist and educator (1953 - 1994)
} 


\section{STRUCTURING OUR RESEARCH EFFORT.}

This research is centred on the primacy of the business process model. We assert that the model has primacy. The model is defined as:

"A description of the system which captures domain specific requirements

expressed within an executable meta-model"

The model is built by taking a consistent view of many issues including enterprise strategic direction, stakeholder intent, risk management and maximisation of efficiency. Simulations can be applied to scope the process and resources required. The system is implemented from the model.

The figure below shows the relationship between the model and the research areas. Emphasis on the model is intended to decouple research into 'bite sized' chunks and avoid fragmented many-to-many research relationships.

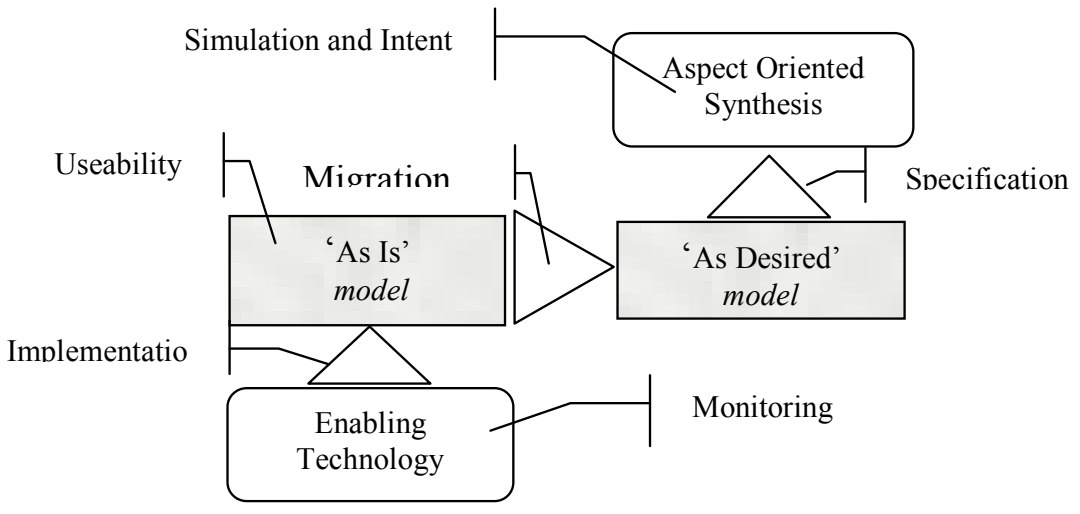

- The 'As Is' model represents the business processes currently performed by the enterprise. In our case, it must be explicit and captured using some formal representation. Often it is implicit in the organisation and not well understood by management. It must be useable to be effective, implying it must present different views to technologists, business analysts, and other roles. These views must all be consistent and add value to the underlying model.

- The 'As Desired' model represents the target set of business processes which the company intends to migrate to.

- 'Aspect Oriented Synthesis' refers to the varying means employed to capture strategic intent and simulate systems in order to specify the 'As Desired' model. Considerations include enterprise strategic direction, stakeholder intent, risk management and maximisation of efficiency. All these considerations and potentially conflicting viewpoints must be synthesised in order to specify a single consistent 'As Desired' model.

- 'Enabling Technology' is the underlying technical infrastructure which 
implements the model. This infrastructure must be capable of executing the model based on the syntax and semantics of the meta-model. The enabling technology may consist of both manual and automated steps. A key characteristic is that only non-functional requirement information can be added at this point.

Statement: Research must relate to the model, its implementation and its metrics.

\section{Take a team approach}

There is a prevailing research culture in many Australian Universities. The culture insists on isolated, individual work; particularly when working towards a $\mathrm{PhD}$. This individuality arises from the mathematical history of algorithmic computer science research.

Unlike many academic areas, research in Business Process Management Systems is a Software Engineering problem. This means

- A breadth of understanding across many academic research areas is required

- Theory must be applied to be understood. Applied theory then acts as a concrete foundation for further research.

- Human factors are paramount.

- Look for leverage, do not re-invent the wheel.

This paper rejects this individualist culture. BPS is a software engineering problem, requiring: both breadth and depth of talent. This breadth combined with limited resources means we must choose research topics wisely so it benefits both the individual and the group. We will take a systematic approach to defining research areas and for each area we will have a thought leader. Areas interact so synergy is achieved, and are constrained to critical issues of general impact, thus guaranteeing active and committed input from the team. An area leader is responsible for academic leadership and exploring inter-area synergy. Publications are expected to be co-authored.

From a $\mathrm{PhD}$ standpoint, this raises the issue of how to assess academic contribution in an environment where the thought leaders ideas in an area may undergo significant revision or indeed lead to novel contributions from within the team. Some Universities, such as the Australian National University, have alternate means of defining PhD's. Initial indications are that satisfactory metrics could be determined based on

- Acknowledgment and attribution of thought leadership by the team and the resulting publications.

- Contributing effort to other papers in a secondary role.

- Industry involvement and case studies.

Statement: We will work in a collegial environment on strategic areas. . An area leader is responsible for academic leadership but is expected to welcome contributions and insights from other members. There is synergy between areas, and we expect robust contribution both from within and across areas. This will lead to many co-authored papers. New avenues to define a PhD will be explored based on existing overseas and Australian approaches. 


\section{Accept we are naive}

Initially, researchers in this area will be naïve, traversing a steep learning curve, and exploring many wrong paths. It is important to have the freedom to move quickly and adopt and discard approaches rapidly. Learning progresses best by taking standpoints and testing ideas. Decisions are forced by milestones on pragmatic projects. This ensures theory and understanding become a concrete foundation for later research.

In the initial phase, papers should be written only for compliance purposes and as an exercise. It is likely many papers will later be contradicted as mistakes are discovered. Later research will be built on the foundation of strong applied knowledge of theory and pitfalls. This research gets us to the leading edge with the tools and experience required to pragmatically structure approaches, assess significance, and understand external contributions. At this point, papers should be significant, publishable in leading journals, and of interest to practitioners.

Statement: We are beginners before we are experts. We create integrated standpoints to be tested by pragmatic application and found wanting. We make progress via our mistakes. Papers are the result of our work, not the objective.

\section{Form Research Alliances}

We require an alliance between process research, decision systems and the application domain.

- Process systems tell us when to take an action or decision, what information exists at this point, and the flow. It acts as the systems integration. It does not tell us how to make a decision.

- Decision support systems tell us how to make choices, apply rules, and negotiate optimal outcomes amongst independent agents within the context of process.

- Application domain. To evaluate and exercise process and decision systems we need an application domains. The application domain is describable by concepts in the other domains..

Statement: We will form alliances with complementary research groups and will lead the process and systems integration effort.

\section{Call in expertise.}

Process provides the context for specialist areas such as security and legacy system integration. When required, we will delegate to the specialist areas rather than become specialists ourselves.

Statement: There exist subsets in the Task-oriented area but outside our area of expertise. Call in this expertise and work in tight collaboration.

\section{Leverage off standards without being paralysed}

There are many competing and evolving standards in this area. There is a high risk of suffering 'analysis paralysis' if we try to correlate standards and understand competing but similar approaches. We will make progress by setting our own objectives and building our own view of how such standards should operate. We can use this knowledge to examine 
various standards and determine both their applicability and their ability to form consistent and complete sets. We will apply them pragmatically to real domain problems. This will give us experience to get a handle on which aspects of standards are important plus their practical human factors and technical issues.

Statement: The diverse and conflicting range of standards will be managed by a requirements driven approach. We will set our own requirements based on our objectives and match to standards as opposed to an exhaustive understanding of all possible current standards.

\section{Contribute to an Open Source project.}

Targeting an open source project provides business, technical, and mentoring benefits. It allows us to make pragmatic progress whilst still referencing emerging standards where possible.

- Business benefits. There will be a commercial battle in the BPS space. Open source has a different group of supporters and has the potential to form an enduring sizeable niche for business development without getting crushed.

- Technical Benefits. Participating in development gives insights into how these systems really work and a framework from which we can develop extensions. Concrete experience will help us define meta-models helping us to understand and evolve standards.

- Peer review. It will provide active external review and mentoring by people to whom pragmatic outcomes matter.

Statement: Our strategic aim is to be a leading research and development group within the Open Source community. We will acknowledge and understand other approaches, but will not allow ourselves to be distracted.

\section{Experiments are required}

Experiments must be conducted for three reasons.

- No system or process is useable unless it supports human factors issues and can cope with the fact that enterprise system development is a 'wicked problem' which cannot be resolved with traditional analytical approaches (Rittel and Webber 1973).

- Experiments force researchers to make pragmatic decisions. These decisions mitigate a tendency to 'analysis paralysis' due to complexity and divergence in this area. These decisions then provide concrete foundations for further research.

- Pragmatic outcomes demonstrate competency and provide a direct link to industry.

Wicked problems cannot be resolved with traditional analytical approaches (Rittel and Webber 1973). They consist of a set of interlocking issues and constraints that change over time, embedded in a dynamic social context. (Poppendieck) Attempting to baseline requirements and then use an analytical approach to reach a solution is a recipe for disaster with wicked problems. These problems are resolved through discussion, consensus, iterations, and accepting change as a normal part of the process. To evaluate the new 
approaches we need to be able to test the whole development process from stakeholder negotiation, through project planning and contracts to final implemented system.

Pragmatic Decisions. There is significant risk of getting into 'analysis paralysis' in this area due to complexity and lack of consistency. By forcing test implementations, researchers will be compelled to make decisions and then experience the results. This will stimulate researchers to propose consistent and viable solutions and also provide a concrete foundation for further research. This foundation will then act as a base to compare competing approaches and a lever to aid understanding.

Statement: We will work in iterations of theory and practice. Theory and research determines our next step, practice and experience clarify and make concrete our concepts

\section{A research laboratory is required.}

This document assumes the existence of a software engineering laboratory. This laboratory must act as a 'process container' to allow different approaches to be efficiently tested and studied. All elements common across projects must be extracted and standardised to ensure we do not waste effort on unimportant issues. The laboratory must also provide support for ongoing generation and retention of intellectual property and provide tools to assist teamwork.

Statement: Progress requires practice based insights and demonstrated competency to test theory. To achieve this we require a properly designed research laboratory. ${ }^{7}$

\section{RESULTS TO DATE}

In the twelve months since this strategy was proposed the following has occurred:

- The Software Effective Process Group has been formed and research begun. The group consists of a systems architect plus specialists in middleware, query languages, testing, and decision systems. Supporting this team are four honours students plus one third year student project group. This gives a total of fourteen people working on interlinked research.

- An alliance is established with the Decision Systems Laboratory www.dsl.uow.edu

- An alliance is established with the Supply Chain Research Centre at the University of Wollongong.

- A research laboratory has been established. This laboratory acts as a 'software process' container and allows target processes to be researched and tested. Tools are also available for knowledge management and teamwork.

- Research laboratory commissioned and is now investigating jBPM. More information is available at www.dlab.uow.edu.au

\footnotetext{
${ }^{7}$ See the working paper on Dynamic Lab. The pilot of DLab exists and is currently evaluating processes.
} 
- Industry partners have been identified and linkage grants are currently under negotiation.

- A series of papers outlining the strategic direction, research areas, research synergy and supporting dynamic laboratory infrastructure have been written. These are available for comment on www.dlab.uow.edu.au

- A major Australian project is utilising a new software development lifecycle for 'system of systems' development which has arisen out of this research strategy. Later papers will report on both the theoretical basis and practical impacts of this work and other research by the group.

There are a number of ongoing challenges. Firstly, a funding Key Performance Indicator catch-22 exists where, in order to be funded, a group must have a track record. Given that the software effective process group is a start up, this situation is not satisfied. There is long term strategic funding available, but pursuing this is a high risk strategy in itself. Secondly involves changing the academic culture. This culture supports individual academic research and impedes team based pragmatic collaboration. This paper proposed an alternate viewpoint to the benefit of both computer scientists and software engineers. The third challenge was somewhat unexpected. Members of the Software Effective Process Group have become in such demand in industry that financial imperatives have led to some members, including this author, joining industry. Every challenge is an opportunity. Provided academic support can be maintained, having some of our team actually in industry presents tremendous leverage for action research and case studies.

\section{CONCLUSION}

This paper presented a framework to conduct software engineering research into process oriented systems within an Australian University. The framework provides a philosophy on how one should conduct such research; recognises the importance of theory and practice; and highlights alliances between faculties, across Universities and with industry. Such a framework is important as it provides an antidote for the short term, operational approach currently encouraged by Australian universities key performance indicators. The direction provided by the framework is proving effective and results are already evident. Significant results will take time, agility is required and much will be learnt through experience. Discussion is welcomed on any aspect of this paper.

\section{REFERENCES}

Broadbent M., M. K., McDonald, M. (2003). "IT success Requires Discipline and Innovation." Gartner FT-20-0693.

Carr, N. G. (2003). "IT Doesn't Matter." Harvard Business Review(May).

Gartner (1997). Nine reasons why IS Organisations Do Not Do BPM.

Hammer, M. and J. Champy (1993). Reengineering the Corporation, Harper Business, New York, NY.

Milner, R. Theoretical foundations of mobile distributed processes and the Pi Calculus.

Poppendieck, M. "Wicked Problems." Software Development Magazine(May 2002). Rittel and Webber (1973). "Dilemmas in a General Theory of Planning." Policy Sciences Vol. 4: pp 155-169. 
Sauer, W. (2003). "Establishing the Business of the Future: The Role of Organizational Architecture and Information Technologies." European Management Journal 21(4): 497-508.

Smith H, F. P. (2003). Business Process Management - the Third Wave.

Smith H., F. P. (2003). IT Doesn't Matter Business Processes Do, Meghan-Kiffer Press.

Smith, P. F. a. H. (2005). "The next generation." BPtrends.

W.M.P van der Aalst, A. H. M. t. H., B. Kiepuszewski, and A.P. Barros "Workflow Patterns."

Zmud, R. (1998). "Editors Comments." MIS Quarterly 28 No. 3: pp. xxxix-xxxii. 